\title{
Rupture and Repair in Mentalization-based Group Psychotherapy
}

Anthony Bateman, Research Department of Clinical, Educational and Health Psychology, University College London, UK; The Anna Freud Centre, London, UK.

E-mail: anthony.bateman@ucl.ac.uk

Chloe Campbell, Research Department of Clinical, Educational and Health Psychology, University College London, UK; The Anna Freud Centre, London, UK.

E-mail: c.campbell@ucl.ac.uk

Peter Fonagy, Research Department of Clinical, Educational and Health Psychology, University College London, UK; The Anna Freud Centre, London, UK.

E-mail:p.fonagy@ucl.ac.uk

Corresponding author: Anthony Bateman 


\begin{abstract}
The paper explores ideas about the role of group mentalizing - the experience of joint attention and shared intentionality- as a process that can support the emergence of more collaborative and salutogenic social functioning. This is based on developmental and evolutionary thinking about the importance of joint attention in human social cognitive development and functioning. The importance of experiencing rupture and repair as part of the process of thinking together - while also working with the separate nature of our thoughts - is described, emphasising that it is through an understanding of the complex and inevitably uneven and challenging nature of joint attention and social cooperation, that such cooperation is itself made possible.
\end{abstract}


This paper seeks to explore the role of group psychotherapy as an environment in which individuals can practice, participate in and contribute to a mentalizing social environment. The complexities of rupture and repair are an essential part of this process: the rough and tumble of human social experience is writ large in such moments, and by ruptures being tolerated, managed and repaired, the possibility of participating in the necessary and ongoing work of social cooperation is opened up for the individuals involved. The context for this exploration is an increasing interest in the role of the wider social environment in understanding psychopathology: the interactional impact of developmental and social challenges that might generate greater vulnerability to disorder, and the role of the social world as an ongoing resilience factor across the life course.

In thinking about this clinically, we have recently described 'three communication systems' associated with effective psychotherapy (the "teaching" and "learning" of content; the emergence of robust mentalizing; re-emergence of social learning outside treatment (Bateman, Campbell, Luyten, \& Fonagy, 2018)) and emphasized the important role that the social environment, beyond the consulting room, plays. In particular, we have noted the importance of the experience of applying the benefits of social learning, acquired in therapy, to the social environment beyond it. In so doing, we have insisted upon the limits of psychotherapy for an individual whose social environment is not sufficiently benign or supportive of mentalization; in other words, it does not support thinking about the mental states - the thoughts, feelings and beliefs - that underpin behavior. Indeed, the family, or social environment, for example in a prison or school, may be distinctly anti-mentalizing with no interest in mental states and organized around coercion and personal threat. Such an environment tends to be one in which there are many ruptures but few repairs. However, we have largely tended to discuss these three communication systems in the context of individual or family therapy. Our purpose here is to apply some of our thinking about the social 
underpinning of psychopathology to group psychotherapy. We will suggest that a group, organized with a primary aim of facilitating mentalizing as part of mentalization based treatment (MBT) (Bateman \& Fonagy, 2016), provides an opportunity - beyond the dyadic or familial environment - for individuals to develop the experience of thinking collaboratively that is necessary for healthier social functioning. We also highlight the importance of ruptures and how they 1) influence the development of mentalization and trust in care deliverers as teachers of culture and 2) how ruptures and repairs in group psychotherapy can facilitate mentalization and re-gain epistemic trust.

\section{Communication as a Culture-propagating Instinct}

The theory of epistemic trust - as applied to developmental psychopathology - takes as its starting-point the idea that as a part of the evolution of our culture-building and transmitting capacity, a particular human social cognitive adaptation has emerged that allows us to fast-track learning, in particular social and cultural learning (Csibra \& Gergely, 2011). To make the learning of such complex and opaque things efficient, "humans evolve specialized cognitive resources that form a dedicated interpersonal system of mutual design in which one is predisposed to 'teach' and to 'learn' new and relevant cultural information to (and from) conspecifics" (Gergely \& Csibra, 2005, p.472). In achieving the challenging task of learning how to successfully navigate their culture, the child is dependent on the reliability and trustworthiness of their teachers/caregivers/elders. The task is easier if the child can accept what their elders tell them about how the world works at face value (Wilson \& Sperber, 2012). This however potentially leaves the learner vulnerable to being misinformed, either accidentally or intentionally; as a protection against this, we have developed the capacity for epistemic vigilance (Sperber et al., 2010). It is suggested that we look for certain characteristic features when someone is communicating with us, which, if present, cause us to 
relax our epistemic vigilance. When epistemic trust is stimulated in this way, we can accept that what we are being told is something known and shared by everyone belonging to our cultural group (Gergely \& Jacob, 2012). These communicational features are known as ostensive cues (Russell, 1940; Sperber \& Wilson, 1995) and form part of the natural human range of teaching behaviors. Ostensive cues include eye contact, calling by name, sensitive conversational turn-taking, and (in infants) the use of motherese (Csibra \& Gergely, 2006; 2009; 2011).

In this way, knowledge about how to function and manage the social world is acquired through a process of "natural pedagogy" (Csibra \& Gergely, 2009). Csibra and Gergely (2009) summarize several intriguing developmental experiments, which give powerful support to the theory of natural pedagogy. For example, in one simple demonstration, 6-month-old infants were shown to follow an agent's gaze shift selectively to an object only if the gaze shift had been preceded by either eye contact with the infant or infant-directed speech (Senju \& Csibra, 2008). Shared attention with an agent is triggered by the infant experiencing the agent's interest. The interest triggers the infant's expectation (epistemic trust) that there may be something relevant for the infant to learn.

\section{Joint Intentionality: The Significance of "We-ness"}

Another line of thinking related to these ides has emerged from the work of Tomasello, who has emphasized the social nature of the human brain, and in particular the significance of 'joint attention' in human social cognition (Tomasello, 2016). Joint attention refers to the ability to focus with another on both external objects, and on mental content - of particular significance is the ability to understand how and why mental states might differ (O’Madagain \& Tomasello, 2019). O'Madagain and Tomasello suggest that it is through the uniquely human interest in "joint attention to mental content" that transforms children's 
mind-reading and reasoning abilities (O’Madagain \& Tomasello, 2019, p.1). An infant who benefits from being effectively mentalized is rich in experiences of joint attention to mental states. As well as feeling intensely rewarding for an infant, such moments of "we-ness" confer the powerful benefit of stimulating epistemic trust and creating an openness to collaborative social learning.

Human sociality is explained by the remarkable capacity we have to imagine the mental states of others - to mentalize. Tomasello points out that the key is the capacity to coordinate perspectives and thus appreciate the distinction between the subjective (one's own view) and the objective (actual physical reality "out there"), and then to coordinate these with knowledge of another individual's mental state: quite a complex triangulation (Tomasello, 2016). We would suggest, along with Tomasello, that a joining of minds is the crucial ingredient of mentalizing. Joint attention defines a common object at the same time as acknowledging that we have different perspectives on it. Identifying the potential for difference is the first and necessary step towards their alignment, which in turn is the critical underpinning for human collaboration.

Tomasello identifies this process as the creation of a "dual level structure" of shared intentionality (Tomasello, 2016, p.46). It is dual level because it encompasses a shared focus on something and individual perspectives upon the same thing (Tomasello, 2016). If we assume that increasing cooperation was the motivator of selective advantage, it is not surprising that we developed a skill that immeasurably advanced our capacity for social coordination and communication. In short, we do better as a group than as an individual. Building on joint attention (Liszkowski, Carpenter, Henning, Striano, \& Tomasello, 2004), we evolved the capacity for sharing minds, for joining together in a single moment of combined attention and an awareness of internal states in the self and in the other. Cooperation is immeasurably advanced by being able to compare and coordinate different 
perspectives on the same situation. Tuomela $(2005$, p.327; 2007, p. 83) has suggested a specific term for this category of actions as "jointly seeing to it." The feeling associated with this joint intentionality, the feeling of "We-ness," may be underpinned by and certainly generates the potential for social collaboration. In the 'we-mode' of social cognition, individuals have the experience of being part of a set of thoughts and feelings that are beyond their own. This sharing of minds in an irreducibly collective mode of cognition has been recognized by many including developmentalists (e.g. Tronick, 2008), by psychoanalysts of most classical schools (e.g. Winnicott, 1956) and increasingly by neuroscientists (e.g. Gallotti \& Frith, 2013).

Learning about culturally transmitted and relevant knowledge first takes places in the context of early caregiving relationships. We suggest that secure attachment is created by a system that is capable of simultaneously generating a sense of safety (Bowlby, 1969) alongside epistemic trust (Fonagy, Luyten, Allison, \& Campbell, 2017a; 2017b). The biological predisposition of the caregiver to respond contingently to the infant's expressive displays creates the foundation for the infant to acquire further knowledge from that individual. During what we have termed "marked mirroring interactions," the attachment figure will "mark" her referential emotion displays to signal the generalizability of knowledge and effectively to instruct the infant about the infant's subjective experience. (Fonagy, et al., 2002; Fonagy \& Target, 2007; Gergely \& Watson, 1996). "Marking” by the caregiver as part of "good enough" mirroring serves as an ostensive cue to the infant that the concurrent mirroring of affect signals is relevant and generalizable.

\section{The culture of a group: Practicing "we-ness"}

Individuals who we tend to treat in MBT have often found the task of joining together with others and engaging with them in a reciprocal and mutually influencing creative mental 
interaction e.g. thinking together, based on a capacity for mentalizing and shared intentionality, has been limited and compromised. The possibility of joint attention to mental content described as so developmentally significant by O’Madagain and Tomasello (2019) may be aversive or unreachable. The task of thinking together, of directing shared intentionality towards exploring mental states, may emerge as a highly dysregulating experience for individuals with personality disorders, for example. MBT - as do other forms of treatment - seeks to explore mental states in a more regulated and contained manner. In so doing, the work of therapy is to introduce the individual to a form of social cognitive cooperation that is essential for effective social function, but which - through its interpersonal and collaborative nature - is particularly complex and hard to sustain. In MBT, the sense of working together - joint intentionality - in relation to mental states is made more or less explicit. The complexity of this task - expressed in the therapist's frank admission of possible mistakes, uncertainty and learning from the patient's communication - is made clear, as is its intrinsic interest and its value when it comes to forging of social connectedness - as expressed in the genuine curiosity and spirit of inquiry that is part of the mentalizing stance.

When it comes to MBT in groups, the multifaceted nature of social communications creates a more complex and mercurial form of joint intentionality. The forming of defensive alliances, for example, may create a sense of thinking together between those individuals, but one which precludes other forms of joint thinking. Any group starts to develop its own 'micro-culture,' based on agreed social mores or traditions. Many of these, particularly at the outset, are inherited culture: rules about timing, seating arrangements and constraints about communication outside the group. As a group develops there will be other more complex and subtle aspects of the group's culture which will be more singular - the "climate" of the group, the position of authority figures, attitudes of deference or otherwise to the group leader, ways of managing interruptions and emotional outbursts. The experience of being a part of, being 
challenged by, and contributing to the culture of this group is a fundamental aspect of MBT because it introduces and regulates the experience of making a cultural contribution. This has a two-fold benefit. The first is to begin to apply some of the mentalizing experiences that are supported in individual psychotherapy (communication system 1) in a wider environment, but one which is more regulated and protected than the patient's natural social environment - it is, one might say, communication system 3 with stabilizers. The second, hoped-for, benefit of the "cultural" experience of MBT group work is that it enables the patients to begin to experience their agency within a group. The validation and acknowledgement of mental states is a vital part of the treatment process in MBT. Experience that recognition of one's subjectivity within a group setting is a powerful social experience that can create a virtuous circle as mentalizing becomes cooperatively reinforced and supported. It can, however, threaten to be a potentially dysregulating experience, and this is why rupture and repair are such a vital part in sustaining a mentalizing culture.

\section{The Role of Rupture and Repair: Developing Joint Intentionality}

Group psychotherapy, and instances of rupture in group psychotherapy, can reveal how epistemic mistrust can generate a vicious cycle of social isolation and social dysfunction. Ruptures in therapy take a number of different forms which can be classified as disagreements about the tasks of therapy, disagreement about the treatment goals, and tensions in the patient-therapist relationship or between patients in a group (Safran \& Muran, 2000). Our purpose here is to consider the ruptures in terms of a break in the reciprocity of mental processes between people and a collapse in embryonic epistemic trust so no learning can take place. The task in seeking to repair these instances revolves around seeking to generate a sense of shared thinking, a "we mode" position, as described above. It is from such an alignment of intentionality that a more virtuous interactional cycle might emerge. 
In MBT groups, a structure is imposed at the start of the group to build the foundations of therapy and to establish agreed upon goals. The leader initiates a number of tasks for the group to undertake with the aim of building resilience in the group and to manage and repair ruptures, which are so common in groups for people with severe personality disorder. Resilience, founded on shared and operationalized principles, maintains social and group cohesion when pressure and tension build and prevents interactions spiralling out of control and getting to the point at which repair is really a desperate attempt to prevent drop out. There are two specific group interventions that are used early in the formation of an MBT group to reduce a sense of isolation and to signify the importance of “we-ness". Both processes generate companionship, shared ownership, and sense of belonging (Johnson, Burlingame, Olsen, Davies, \& Gleave, 2005). The processes are also triggered at intervals throughout the group particularly when ruptures occur or impasses are apparent. One principle that MBT clinicians follow is to intervene always with a long-term aim of creating the dual level of intentionality discussed earlier in which there is an individual level focus whilst simultaneously each member has to take a shared perspective leading to an agreed outcome which is different from the personal and creates an experience of being part of something that is beyond the sum of its parts. The structured tasks at the start of an MBT group are:

1. Values driven group discussion

2. Formulation of the group by the group in relation to individual formulation.

\section{Values Driven Group}

MBT has an introductory phase which to a large extent addresses the processes required to facilitate Communication System 1. At the end of this phase, patients have considerable information about personality, attachment, mentalizing, their significance in the 
formation of their problems, and their personal formulation. At the start of an MBT group, the clinician is first tasked to generate the agreed values of the group in terms not only of the structural aspects as mentioned earlier but also in terms of their attitudes to themselves and to each other. It is not left to the group to develop over time. The clinician leads the discussion with the primary aim of stimulating shared values that all can relate to. To facilitate this process, the clinician has a list of possible values; many clinicians and most patients have difficulties in identifying values, let alone deciding which they might own jointly and give priority to. For example, a central group value that the clinician may propose is that "we create a sense of fairness (clinician writes up fairness on the board). What I am thinking about is how we are fair about listening to others and seeing things from their point of view and how we are fair in talking about ourselves and having others see things from our point of view?" Another example might be tolerance - "will we respect diverse views or try to insist people see our own perspective and agree with us?" Gradually the group develops a series of values which are carefully defined and recorded and referred to as the group progresses.

For each proposed value, the group discusses three specific questions, all of which facilitate mentalizing in the group:

- Is this value important to me or do other people think that it is important, and I should see it as important?

- If we agree it is important, how will we decide if it is being followed? What are the indicators of intolerance, for example, or unfairness?

- Does this joint value play a role in my life at the present time?

The second interventional process to stimulate group culture and initiate agency and judicious use of epistemic vigilance in place of epistemic distrust is through formulation of the group, by the group. Again, MBT is concerned with the process of generating the shared formulation just as with the formation of shared values, rather than the final outcome; it is the sense of 
working together to create something jointly that all have contributed to that is of significance for mentalizing as part of the driver of learning about oneself from others.

\section{Formulation of the Group by the Group}

In MBT all patients entering the group have a personal formulation collaboratively developed with the clinician (Bateman \& Fonagy, 2016). This mentalizing formulation is structured in form and agreed in writing with the patient. Importantly, the patient has discussed the attachment strategies that are activated when under personal stress and they are asked to present this aspect of their formulation in the group when they start. All patients currently in the group are asked to present their own formulations to the new member when they introduce themselves and then the new member does the same - this follows a group value of "tolerance," for example, which includes welcoming new ideas and new people and learning from them without prior judgement. Whilst this requirement to present one's own formulation is personally exposing, it also provides an immediate immersion into relational culture for learning about oneself and others. It is a prelude to the more complex task of the group - deciding what sort of group they are. This work is implemented by the clinician who specifies the task - "we need to review the group and consider what sort of group we have become and think about whether we want to change that - are we maintaining our values or not?” Indicators to instigate this task are when a new patient joins, an impasse in the group, a failure to maintain the group values, a decision to re-orientate the group process, or when interpersonal ruptures occur. It is the bedrock of repair of the group as it establishes joint intentionality. 


\section{Rupture and Repair}

Somewhat schematically simplifying a process of repair, we might break this into three stages:

1) Accept full agency - the therapist/group leader nondefensively identifies their role in the emergence of the rupture, specifically when the rupture is between them and a patient. But it also applies when the rupture is between two group members.

2) Empathy and validation - the therapist/group leader acknowledges and shows empathy and understanding about the impact of the situation on the client's state of mind.

3) Make the client agentic - the therapist/group leader turns to the client to think together about repair.

\section{Clinical Example: Member Rupture with the Leader}

This example took place in a weekly outpatient group for patients with borderline personality disorder. The group work was part of an intensive 18-month out-patient MBT program for patients with BPD with extensive co-occurring conditions,.

Nigel was a 26-year-old man who was self-destructive and constantly getting into arguments with others that resulted in violence. He had been arrested on a number of occasions. He recognized that his temper was out of control and stated that he wanted to do something about it. In the assessment, a number of violent episodes were explored with him. One aspect seemed to stand out - most of his arguments began by him stating that he wanted something from someone who then failed to give it to him. This ranged from a request to a friend to borrow some money to demands to stay overnight with people. This was embedded in his formulation as an attachment process in which he seeks proximity to someone for a need he has, only to find that he 
is rejected, which makes him feel worse. This results in him trying to get what he needs by forcing the person to give it to him. However, currently this pattern involved his constant requests to his former girlfriend and to social services to visit his daughter. All requests were declined, and in response he threatened them on the phone and during meetings. In the group, he voiced his frustration and anger with his girlfriend and the social services.

On one occasion he arrived a few minutes before the end of the group and requested some time to talk about recent events with social services. As the group was nearly at an end, the clinician said that there was very limited time so perhaps Nigel could summarize quickly or even wait until the following week.

Nigel: Oh, I see. So you think there is no time, do you? Well, I will make sure that there is time. I am not leaving the group until we have discussed it. So, you sit down and listen to me. (Nigel experiences a rupture and as though the leader and group do not want to hear him).

Other group members stated that they could stay for a time: "We have got a bit of time so why don't we go on for a bit?"

Leader: So let's try to use what time we have left. (Leader tries to repair the rupture by giving Nigel time in the group but fails to do so because he has not implemented the first step of repair, accepting responsibility for creating the rupture, and tries to implement a quick solution which continues to indicate implicitly that there is limited time for any serious discussion.)

Nigel: You are not interested. The only thing you want is to get out of here. You don't care about our problems. Fuck you. (Nigel is defensive and expresses hostility and malevolence onto the leader seeing him as selfish and only concerned with his own needs). 
John: He is at least staying to listen, so what happened? (Trying to repair the rupture by helping Nigel see the leader in a different light. Trying to help Nigel accept the leader as someone who can listen. There is now some triangulation - Nigel holding his own perspective, John suggesting another perspective, and an offer of listening to the story without pre-judgement with other members of the group listening. This is a first step in supporting Nigel to move from a singular to a multi-faceted perspective as well as reducing his tension. The rupture with the therapist remains).

Nigel: Social services won't let me see my daughter. They say I am threatening and won't be good for her. She is my daughter. I should be able to see her. I went yesterday and they threw me out. I am going back to sort them out. (Nigel shares a painful experience of being perceived as dangerous and not being able to see his child.)

Leader: Nigel, can I say something here. Is this about your asking them again after you have tried really hard to manage your feelings and then being rejected, with them not understanding your honest wish to see your daughter? (Now the leader is more able to use step 2 empathizing with Nigel's underlying feelings of honest care for his daughter and his sense of being misperceived. This is empathic to Nigel's perspective, reduces tension further and allows Nigel to shift from defensive anger to more curiosity. The rupture with the therapist is bridged although not yet repaired).

Nigel: (A little calmer after receiving this validating statement) Yes. I am going to get them and make them listen.

Leader: Can you tell us a bit more about your wish to see your daughter and what you have in mind in seeing her? (Here the clinician is trying to explore Nigel's positive attitude towards himself to increase his self-esteem, along with his feelings for his relationship with his daughter rather than heighten his feelings of being 
misunderstood by social services which is more likely to increase his agitation at this moment.)

Nigel: Why can they not see that I am not dangerous towards my daughter? I have never harmed her or lost my temper with her. They don't listen and they don't give a shit.

John: They always try to protect themselves, so they always play safe. It is easier for them.

The clinician now intervenes because John is no longer triangulating perspectives as he was earlier, which might build joint intentionality, rather he is collapsing into collusion with narrow beliefs about social worker motives.

Leader: Before we get to that I was thinking that - didn't this just happen here? You wanted to talk to us and for us to see what was going on for you and my first response was to tell you we did not have time (here the clinician is trying to engage in step 1 which is to non-defensively accept responsibility for the rupture). You felt I did not give a shit and were going to make us listen by keeping us here. (Therapist tries to link what is happening in the session to the activation of an interpersonal process, actually a dominant attachment strategy to manage hyperactivation of attachment, that is central to how Nigel manages rejection and feelings of being misunderstood). Nigel: Well, you are listening now.

Leader: Yes. I want to thank John though for picking it up. John can you talk to Nigel about what you were picking up from him in all this.

A little later in this discussion the leader brought up an element of the problem that had been considered in the group before.

Leader: Maybe the problem is now that we have been forced to talk with you and so it is not quite the same as if we had given it freely. 
Rupture and Repair in Mentalization-Based Group Psychotherapy

Nigel: I don't care about that.

Leader: Are you sure? It brings to my mind your sense that what you want is for people to "really want" to help you but that the only help you get is when you force people to give it. Can we think about that? (Here the clinician returns again to the formulation which includes Nigel's wish for proximity seeking especially when he experiences stress which is unrecognized and his reactivity when the responsiveness is dismissive. The collaborative development and sharing in the group of the initial formulation sensitises Nigel to "listen" to the clinician and other members if the group because a channel through epistemic vigilance is now open).

In this discussion, Nigel became calmer and less defensive when the leader was able to empathize with his anger at being misperceived. This allowed the clinician to take up the attachment process that had been enacted. In essence Nigel had made a request to the clinician, with whom he had a positive alliance, and this had been rebuffed. This triggered his pathological rigid attachment processes as he struggled to maintain emotional stability: he attempts to control the other person through threats in order to manage his experience of rejection and loss of control. This illustrates how rapidly the epistemic channel can close once insecure and dismissive and disorganized attachment strategies are activated and a rupture appears. Insecure attachments leave mentalizing processes vulnerable to being lost under stress which means the main driver stimulating epistemic interest about oneself from others shuts down; the channel seeking perspectives from others closes. At the same time the clinician's "listening" channel was also reduced due to his low mentalizing created by some irritation about Nigel being so late and disrupting the group. In his response the clinician retreated to real world reality by pointing out that there was not much time left, thereby reducing any interest in Nigel's mental states, which itself reduces epistemic trust. Inevitably 
this increased Nigel's attempts to control him. It was a clinical error which triggered Nigel's habitual response pattern, illuminating his interactions with others. This had been identified in the assessment and was now being activated in the group process. The clinician correctly tried to reduce his arousal and then start exploring the relational process, developing an alternative perspective about what was happening. Importantly, other group members need to join the discussion if there is to be achievement of "we-ness" from the exploration of both Nigel's subjective experience of the incident he describes and of his experience of others in the group. The aim of the MBT clinician is to reach a point through exploration of mental states where Nigel's experiences a feeling that his subjective sense of self has been recognized and others have contributed to that process, and in doing so the group have opened a channel for learning together. In the end the group managed to clarify that Nigel had wanted to express his desperation but was unable to set up a facilitating context to do this. The result was that Nigel found himself rebuffed, leading to his natural coercive response. After this pattern was defined again and related to his formulation, it was possible to explore his sense that once he had forced people to do things it was ineffective in meeting his need because it was not given freely. This was worked on later in further groups and his individual sessions.

\section{Clinical Example: Member Rupture with Member and Group Rupture with Leader}

A group of six patients, four female and two male, with severe borderline personality disorder had been meeting for 4 months. The group members identified strongly with each other. All engaged in self harm, had been in hospital for suicidality, and had shared their formulations at the start of the group openly which showed that they all had similar interpersonal and social problems. One of the values they had agreed upon after considerable discussion was 
summarised as "self-reflect when reacting," e.g. look before you leap/self-observation whilst talking.

A member, Andrea, reported that she didn't know what to do about her boyfriend. She wanted to be with him, but he was avoiding her and it made her feel unwanted and unattractive. As she talked about this she became increasingly upset. The group members tried to reassure her that it would be OK and gave her all sorts of suggestions about how to deal with the problem. Each time she responded by saying their comments were unhelpful. Suddenly another patient, Catherine, said "Oh stop moaning for goodness sake. Leave him then so that we can talk about something else. You are being pathetic." Andrea began sobbing. This was a rupture in the group and has the potential to shut down epistemic trust in the group.

The explosiveness of this comment froze the group who were taken aback by the ferocity behind Catherine's remarks and they naturally turned and looked at the group leader. The group leader felt compelled to respond, initially to reduce the anxieties and tension which is necessary if a rupture is to be addressed and managed constructively. He immediately implemented an MBT "stop and stand" and "siding" procedure to prevent escalation of the interaction. In these MBT interventions used in groups for people with severe personality disorder the leader controls the process of the group by taking over (Bateman \& Fonagy, 2016; Bateman, Kongerslev, \& Bo Hansen, 2019). They do not wait for someone to respond to see how they manage the tension - high tension in people with BPD undermines their mentalizing and so creates reliance on low level mental processing systems leading to self or other destructive behaviors. Siding involves giving clear support to the most vulnerable party of a rupture. In this situation it looked like it was Andrea. 
Leader: Hang on. That was a bit sharp. What are you saying here? (looking at Catherine). Andrea is struggling with how to manage her boyfriend and talking about how she feels hopeless, and you say this is pathetic. That sounds awfully judgemental to me (turns to Andrea). Andrea, I don't think this is pathetic. The problem we have is how to help you to feel a bit stronger about it and that is our problem. We do not seem to have been able to do that yet. (Making the issue one every group member addresses and supporting Andrea whilst recognising the transactional problem between Andrea, Catherine and possibly other group members).

Leader: Now, Catherine, can you say what it is that is going on for you about this rather than being somewhat dismissive of Andrea?

At this point the clinician has taken the side of Andrea and pushed the focus back to Catherine. This will reduce Andrea's stress and foster more trust in the leader, but it does have the potential to injure Catherine. Now the clinician has to monitor the effect on Catherine as she, too, may become defensive or vulnerable. Indeed, in this group she did so and reacted by challenging the clinician. The siding manoeuvre supporting Andrea was leading to a rupture between the leader and Catherine.

Catherine: You always support her. You like her better. We spend so much time on Andrea in every group that there is nothing for the rest of us and it has no effect. She always says that things we say are useless.

Leader: You may be right about some of that and I am trying to support Andrea. I think it is important that we don't dismiss Andrea and her struggle to work out what to do. I suppose I am "liking" her better at the moment in that sort of way. Maybe it might help if you could talk about what effect Andrea was having on you so we can understand what fuelled your comments so strongly. 
Rupture and Repair in Mentalization-Based Group Psychotherapy

The leader is now trying to address the immediate rupture between him and Catherine which to some extent has been formed out of his support for Andrea, so he takes agency for creating this additional difficulty.

It is necessary at this point for the leader to ensure that Catherine talks about herself and does not revert to talking about Andrea. The aim of the intervention is to increase scrutiny of internal self-state rather than the state of others. So the leader was ready!

Catherine: Well Andrea spends all her time

Leader: Catherine before we go back to Andrea can you say more though about what your own experience is, what was happening in your feelings rather than your experience of Andrea.

I was thinking about our value of look before you leap, and in this case, and I don't mean this critically, let's do a look after you have jumped.

Catherine: (Smiles slightly). Well I feel frustrated and I hate the idea that I can do nothing to help someone. I feel so not heard and invisible which is shit.

The tension is now lowered and the rupture between the leader and Catherine is repaired to some degree and can be explored more later, perhaps in relation to favouritism since another value that had been agreed in the group was summarised as 'equality.' Catherine feels there is no equality in terms of time taken or leader interest in each person. This value can be brought in by the clinician to focus the discussion to generate a more robust repair of the rupture. Focusing around a value in this way as the framework for repair supports mentalizing which in turn opens the epistemic channel further.

The conversation continued and as the tension reduced more the clinician passed the focus back to Andrea, no longer taking sides. 
Leader: Andrea, while I can see that what Catherine said was hurtful, what do you make of this sense that every time the group try to help that it seems to go nowhere? Is that your experience?

The group then began to focus on the problem of trying to help someone but that person not finding the support helpful. The problem was that Andrea's negativity gradually produced frustration in other members and left them feeling hopeless, a response that was normalized by the group leader. Nevertheless, the group members were tasked by the clinician to make sure that if they were becoming frustrated when talking to Andrea they should say so. Andrea was asked to monitor her negativity and to identify anything that she found helpful, so that she could let the group know. The leader is trying to generate a process in which all members of the group are engaging with "we-ness" in terms of looking at the reciprocity and interaction of mental process around an interpersonal focus, thereby creating the possibility of each person (in this case Andrea, Catherine and the leader primarily) distinguishing between their own subjective experiences in an interaction and those of others, and the objective reality "out there" of a non- responsive boyfriend and the other objective reality of a breakdown in the values held by the group. By experiencing how another individual's mental state is in motion whilst holding onto one's own, separate state, it becomes possible to differentiate rather than elide them and to see perspectives - a complex triangulation but one that is necessary for opening the epistemic channel and forming 'weness' within the group. Andrea was closed to the support and advice from others in the group and was epistemically distrustful, which is in itself rejecting and rapidly induces others to close off themselves. In the anger and frustration and unkindness none of this can be addressed, so the group leader is tasked with trying to rekindle interpersonal listening - that is mentalizing between members - to open up the epistemic channels. Actively reducing 
anxiety, separating the immediate protagonists and taking some agency for what has happened are the first steps to repair an interpersonal rupture. Using a mentalizing stance to explore the mental states of Catherine and Andrea that are fuelling the ruptures is a prelude to generating a wider discourse in the whole of the group as three of the other patients present had not yet contributed. If this were unaddressed, a further rupture might occur between Andrea/Catherine and the rest of the group. Again, the leader can use the values agreed in the group if one is relevant as the objective agreement 'out there' (perhaps written up on a board in the group room) around which different perspectives are explored.

\section{Conclusions}

Group psychotherapy provides a valuable environment for focussing on the mental state of oneself and others. It can generate complex emotional and interpersonal interactions, which can be harnessed for patients to explore their subjective understanding of others' motives while reflecting on their own motives. Group psychotherapy can also be a challenging area of treatment, with a patient having six to nine minds in the room to think about - the demands of such a sophisticated level of mentalizing almost inevitably causes heightened affect, attachment dysregulation, rupture. The risk is that non-mentalizing interaction and discourse becomes the default for the group. The carefully managed structure of the MBT group and the role of the group leader as a manager for resetting mentalizing are fundamental to MBT practice to prevent iatrogenic group functioning, and to establish a process whereby mentalizing is re-established, and ruptures are indeed repaired. This involves the scrupulous attention of the group leader on the mentalizing processes at work.

One of the desired outcomes of maintaining and restoring mentalizing in the group is the generation of epistemic sensitivity and trust. The focus is on developing a mentalizing culture by increasing mentalizing in all its dimensions; stable mentalizing is the driver of 
learning from others, a process in which people with severe personality disorder have necessarily, from experience, become wary. As a result, they are unable to develop or maintain social relationships and social systems that can further support them. It is the task of an MBT group to support mentalizing to a level of "we-ness" through which group members begin to trust social processes where experiences are shared and become meaningful beyond the individual. This process commences right from the start of the group when patients have to generate their own agreed values that are to be followed by the group and work together to formulate the current aims and anxieties of the group. The hope is that a virtuous circle emerges where joint thinking stimulates epistemic trust and allows learning from others to shape change.

Having initially been developed as a part of an MBT programme alongside individual treatment, Group MBT has also grown as a standalone treatment for BPD in its own right. The use of MBT group psychotherapy across a wider range of diagnoses is an area of further development that has begun to unfold, and we hope will continue. Again, originally developed for the treatment of patients with borderline personality disorder, group MBT psychotherapy has since been developed for individuals with antisocial personality disorder and has also been integrated into work with adolescents and for individuals with eating disorders. The role of epistemic trust and the outcomes for group MBT is an area that would benefit from direct empirical research.

\section{References}

Bateman, A., Campbell, C., Luyten, P., \& Fonagy, P. (2018). A mentalization-based approach to common factors in the treatment of borderline personality disorder. Current Opinion in Psychology, 21, 44-49. doi:10.1016/j.copsyc.2017.09.005 
Bateman, A., \& Fonagy, P. (2016). Mentalization based treatment for personality disorders: a practical guide. Oxford: Oxford University Press.

Bateman, A., \& Fonagy, P. (2016). Mentalization-based treatment for personality disorders: A practical guide. Oxford, UK: Oxford University Press.

Bateman, A., Kongerslev, M., \& Bo Hansen, S. (2019) Group therapy for adults and adolescents. In A. Bateman \& P. Fonagy (Eds.), Handbook of Mentalizing in Mental Health Practice (2nd ed., pp. 129-147). Washington, DC: APPI.

Bowlby, J. (1969). Attachment and loss, Vol. 1: Attachment. London, UK: Hogarth Press and Institute of Psycho-Analysis.

Csibra, G., \& Gergely, G. (2006). Social learning and social cognition: The case for pedagogy. In M. H. Johnson \& Y. Munakata (Eds.), Processes of change in brain and cognitive development. Attention and Performance XXI (pp. 249-274). Oxford, UK: Oxford University Press.

Csibra, G., \& Gergely, G. (2009). Natural pedagogy. Trends in Cognitive Sciences, 13(4), 148-153. doi:10.1016/j.tics.2009.01.005

Csibra, G., \& Gergely, G. (2011). Natural pedagogy as evolutionary adaptation. Philosophical Transactions of the Royal Society of London. Series B, Biological Sciences, 366(1567), 1149-1157. doi:10.1098/rstb.2010.0319

Fonagy, P., Gergely, G., Jurist, E., \& Target, M. (2002). Affect regulation, mentalization, and the development of the self. New York, NY: Other Press.

Fonagy, P., Luyten, P., Allison, E., \& Campbell, C. (2017a). What we have changed our minds about: Part 1. Borderline personality disorder as a limitation of resilience. Borderline Personality Disorder and Emotion Dysregulation, 4, 11. doi:10.1186/s40479-017-0061-9 
Fonagy, P., Luyten, P., Allison, E., \& Campbell, C. (2017b). What we have changed our minds about: Part 2. Borderline personality disorder, epistemic trust and the developmental significance of social communication. Borderline Personality Disorder and Emotion Dysregulation, 4, 9. doi:10.1186/s40479-017-0062-8

Fonagy, P., \& Target, M. (2007). Playing with reality: IV. A theory of external reality rooted in intersubjectivity. International Journal of Psychoanalysis, 88(Pt 4), 917-937.

Gallotti, M., \& Frith, C. D. (2013). Social cognition in the we-mode. Trends in Cognitive Sciences, 17(4), 160-165. doi:10.1016/j.tics.2013.02.002

Gergely, G., \& Csibra, G. (2005). The social construction of the cultural mind: Imitative learning as a mechanism of human pedagogy. Interaction Studies, 6, 463-481. doi:10.1075/is.6.3.10ger

Gergely, G., \& Jacob, P. (2012). Reasoning about instrumental and communicative agency in human infancy. In J. B. Benson, F. Xu, \& T. Kushnir (Eds.), Advances in child development and behavior. Vol 43: Rational constructivism in cognitive development (Vol. 43, pp. 59-94). Waltham, MA: Academic Press/Elsevier.

Gergely, G., \& Watson, J. S. (1996). The social biofeedback theory of parental affectmirroring: The development of emotional self-awareness and self-control in infancy. International Journal of Psycho-Analysis, 77(Pt 6), 1181-1212.

Johnson, J. E., Burlingame, G. M., Olsen, J. A., Davies, D. R., \& Gleave, R. L. (2005). Group climate, cohesion, alliance, and empathy in group psychotherapy: Multilevel structural equation models. Journal of Counseling Psychology, 52(3), 310-321. doi:10.1037/0022-0167.52.3.310.

Liszkowski, U., Carpenter, M., Henning, A., Striano, T., \& Tomasello, M. (2004). Twelvemonth-olds point to share attention and interest. Developmental Science, 7(3), 297307. doi:10.1111/j.1467-7687.2004.00349.x 
Luyten, P., Campbell, C., Allison, E., \& Fonagy, P. (2020). The mentalizing approach to psychopathology: State of the art and future directions. Annual Review of Clinical Psychology.

O’Madagain, C., \& Tomasello, M. (2019). Joint attention to mental content and the social origin of reasoning. Synthese. doi:10.1007/s11229-019-02327-1

Russell, B. (1940). An inquiry into meaning and truth. London, UK: Allen \& Unwin.

Safran, J. D., \& Muran, J. C. (2000). Resolving therapeutic alliance ruptures: Diversity and integration. Journal of Clinical Psychology, 56(2), 233-243. doi:10.1002/(SICI)10974679(200002)56:2<233::AID-JCLP9>3.0.CO;2-3

Senju, A., \& Csibra, G. (2008). Gaze following in human infants depends on communicative signals. Current Biology, 18(9), 668-671. doi:10.1016/j.cub.2008.03.059

Sperber, D., Clement, F., Heintz, C., Mascaro, O., Mercier, H., Origgi, G., \& Wilson, D. (2010). Epistemic vigilance. Mind and Language, 25(4), 359-393. doi:10.1111/j.1468-0017.2010.01394.x

Sperber, D., \& Wilson, D. (1995). Relevance: Communication and cognition (2nd ed.). Malden, MA: Blackwell.

Tomasello, M. (2016). A Natural History of Human Morality. Cambridge, MA: Harvard Univ Press.

Tronick, E. Z. (2008). Emotional connections and dyadic consciousness in infant-mother and patient-therapist interactions: Commentary on paper by frank m. Lachmann. Psychoanalytic Dialogues, 11(2), 187-194. doi:10.1080/10481881109348606

Tuomela, R. (2005). We-Intentions revisited. Philosophical Studies, 125, 327-369.

Tuomela, R. (2007). The Philosophy of Sociality: The Shared Point of View. Oxford: Oxford University Press. 
Verhage, M. L., Schuengel, C., Madigan, S., Fearon, R. M., Oosterman, M., Cassibba, R., .. . van IJzendoorn, M. H. (2016). Narrowing the transmission gap: A synthesis of three decades of research on intergenerational transmission of attachment. Psychological Bulletin, 142(4), 337-366. doi:10.1037/bul0000038

Wilson, D., \& Sperber, D. (2012). Meaning and relevance. Cambridge, UK: Cambridge University Press.

Winnicott, D. W. (1956). Mirror role of mother and family in child development. In D. W. Winnicott (Ed.), Playing and reality (pp. 111-118). London, UK: Tavistock.

Zeegers, M. A. J., Colonnesi, C., Stams, G. J. M., \& Meins, E. (2017). Mind matters: A metaanalysis on parental mentalization and sensitivity as predictors of infant-parent attachment. Psychological Bulletin, 143(12), 1245-1272. doi:10.1037/bul0000114 